\title{
Capturing adverse events in elective orthopedic surgery: comparison of administrative, surgeon and reviewer reporting
}

\author{
Katie Garland, MD \\ Brian P. Chen, MD \\ Stephane Poitras, $\mathrm{PhD}$ \\ Eugene K. Wai, MD \\ Stephen P. Kingwell, MD \\ Darren M. Roffey, PhD \\ Paul E. Beaulé, MD
}

This work was presented at the Canadian Orthopaedic Association annual meeting held in Québec, Que., Canada, June 16-19, 2016

Accepted June 11, 2019

\section{Correspondence to:}

P.E. Beaulé

CCW 1646

Division of Orthopaedic Surgery

University of Ottawa

The Ottawa Hospital

501 Smyth Rd

Ottawa ON K1H 8L6

pbeaule@toh.ca

DOI: $10.1503 /$ cjs.019117

\section{SUMMARY}

Ensuring adverse event (AE) recording is standardized and accurate is paramount for patient safety. In this discussion, we outline our comparison of AE data collected by orthopedic surgeons and independent clinical reviewers using the Spine Adverse Events Severity System (SAVES) and Orthopedic Surgical Adverse Events Severity System (OrthoSAVES) against AE data recorded by hospital administrative discharge abstract coders. In 164 spine, hip, knee and shoulder patients, reviewers recorded significantly more AEs than coders, and coders recorded significantly more AEs than surgeons. The AEs were recorded similarly by reviewers using SAVES and OrthoSAVES in 48 spine patients. Despite our small sample size and use of different AE tools, we believe it is important to highlight that coders, surgeons and reviewers recorded AEs differently. While further investigations on its utility and cost-effectiveness are necessary, we assert that it is feasible to use OrthoSAVES to prospectively record AEs across all orthopedic subspecialties.

E nsuring adverse event (AE) recording is standardized and accurate can bring about improvements in patient care, and may help hospitals track quality-improvement indexes, leading to improved postoperative outcomes and reduced expenditures. However, there is still no one method, tool or protocol that is effective at capturing all AEs.

The Spine Adverse Events Severity System (SAVES) has been successfully implemented to record AEs in spine surgery. ${ }^{1}$ The Orthopedic Surgical Adverse Events Severity System (OrthoSAVES) is a modification of SAVES that has been used to record AEs in general orthopedic surgery procedures. ${ }^{2}$ By using SAVES and OrthoSAVES, we previously showed that independent clinical reviewers reported more AEs than orthopedic surgeons in elective orthopedic surgical patients. ${ }^{3}$

Currently, AEs are often captured by hospital administrative discharge abstract coders via a retrospective review of physician directives in medical charts after discharge. This can be inefficient and incomplete owing to the nature of the coding, and can often not be linked back directly to the chart itself. ${ }^{4}$

In this discussion we outline our comparison of $\mathrm{AE}$ data recorded by orthopedic surgeons and independent clinical reviewers using SAVES and OrthoSAVES against AE data recorded by hospital administrative discharge abstract coders as well as our assessment of the appropriateness of using OrthoSAVES across the general orthopedic surgery population, including spine patients.

\section{WHAT WE DID}

All elective orthopedic surgical patients operated on by 1 of 6 surgeons (3 spine, 1 hip, 1 knee, and 1 shoulder) over a 10 -week period at a tertiary-care teaching hospital were included. Emergency and oncology 
cases were excluded. Surgeons used SAVES (spine) and OrthoSAVES (knee, hip, and shoulder) to prospectively record AEs. No specific annotation was made by the surgeons on the medical charts with regards to recording AEs.

Two independent clinical reviewers with access to electronic records, medical charts and allied health professionals prospectively recorded AEs using SAVES (spine) and OrthoSAVES (knee, hip, shoulder and spine). Reviewers and surgeons were blinded and noncommunicative.

Hospital administrative discharge abstract coding data, recorded by coders via a review of medical charts after discharge, were matched against ICD-10 codes analogous to SAVES and OrthoSAVES. Coders were not directly involved in patient care or the completion of SAVES and OrthoSAVES forms by reviewers or surgeons.

The AEs were recorded by reviewers in spine patients using both SAVES and OrthoSAVES. We analyzed the suitability of each AE tool by identifying any outliers that had to be inputted under the "Others" section on either form (Appendix 1, Table S1, available at canjsurg. $\mathrm{ca} / 019117-\mathrm{a} 1)$.

\section{WHAT WE FOUND}

There were 164 patients included: 48 spine, 34 knee, 51 hip, and 31 shoulder (Appendix, Table S2). Reviewers recorded 99 AEs in 57 patients, which was significantly more than the $31 \mathrm{AEs}$ in 17 patients recorded by the coders $(p<0.001)$. Coders, in turn, recorded significantly more AEs than surgeons, who recorded 14 AEs in 12 patients $(p=0.01)$. Only in 1 patient was the recording of AEs the same among coders, surgeons and reviewers. Overall, 25 AEs were recorded by coders but not surgeons, and 15 AEs were recorded by coders but not reviewers or surgeons (Appendix 1, Table S3). In 48 spine patients, there were 3 AEs recorded by reviewers that were unique to OrthoSAVES (i.e., serous wound drainage, fall and urinary retention) that were recorded under "Others" in SAVES.

\section{WHAT DOES IT ALL MEAN?}

Prospective data collection is time-consuming, which can lead to underreporting of AEs and, hence, the continued appeal of using a retrospective hospitalbased recording system. Whether surgeons and hospitals can rely on hospital administrative discharge coding for accurate and thorough AE reporting is currently equivocal.5,6 Overall, our findings are comparable with those from previous studies, wherein reviewers using SAVES and OrthoSAVES recorded more AEs than coders. ${ }^{7-9}$
In our assessment, we found a significant discrepancy in AE reporting among coders, surgeons and reviewers. Notably, coders recorded 15 AEs in 10 patients that were not recorded by surgeons or reviewers. Upon re-examination, the additional AEs recorded by coders appeared to be based on information written in the progress notes by nurses or other allied health team members, as opposed to notations provided by surgeons. This contradicts the coders' mandate of utilizing only physician directives as their sole source of information, and serves to highlight one of the key limitations with administrative recording. It also underscores the importance of proper documentation by physicians themselves.

A survey of physicians found several contributory factors for potentially underreporting AEs. These included cases in which no harm came to the patient and surgeons did not feel that an AE had occurred, and situations in which $\mathrm{AE}$ reporting was considered to be too difficult and/or time-consuming. ${ }^{10} \mathrm{~A}$ recent commentary also mentions "professional shaming" and the need for qualitative analyses that can add insight into why surgeons may feel it is not necessary to report the more frequent minor AEs. ${ }^{11}$ It is essential that we simplify the process of prospectively recording AEs and make the tools used to record AEs as applicable as possible to a wide range of specialties.

Although SAVES was developed specifically for use in spine surgery, implementing multiple AE tracking instruments within a hospital department is not feasible. Given only 3 additional AEs were recorded using OrthoSAVES compared with SAVES in 48 spine patients, we conclude that OrthoSAVES can be used to record AEs in spine patients; thus, it is feasible to use OrthoSAVES to prospectively record AEs across all orthopedic subspecialties.

We acknowledge our small sample size and utilization of different AE tools. We realize that surgeons filling out SAVES and OrthoSAVES forms may have altered their discharge summaries and included reference to an $\mathrm{AE}$, thereby changing the information normally available to hospital administrative discharge abstract coders. However, anecdotally, this occurred infrequently owing to the presence of significant discrepancies in AEs recorded by the surgeons and coders.

\section{OUR OPINION}

Hospital administrative discharge abstract coders recorded more AEs than orthopedic surgeons, but both recorded fewer AEs than independent clinical reviewers. Using reviewers to track AEs may be preferable, but additional costs related to their employment may be a barrier. Alternatively, changing the culture around the reporting of AEs, as well as training 
surgeons and trainees to better utilize OrthoSAVES, may be more sustainable, especially if it is predicated as a long-term cost-saving measure and qualityimprovement initiative.

Affiliations: From the Faculty of Medicine, University of Ottawa, Ottawa, Ont., (Garland, Chen, Wai, Kingwell, Beaulé); the School of Rehabilitation Sciences, Faculty of Health Sciences, University of Ottawa, Ottawa, Ont. (Poitras); the Division of Orthopaedic Surgery, Department of Surgery, University of Ottawa, The Ottawa Hospital, Ottawa, Ont. (Poitras, Wai, Kingwell, Beaulé); the uOttawa Combined Adult Spinal Surgery Program, The Ottawa Hospital, Ottawa, Ont. (Wai, Kingwell, Roffey, Beaulé); and the Clinical Epidemiology Program, Ottawa Hospital Research Institute, Ottawa, Ont. (Wai, Kingwell, Roffey, Beaulé).

Competing interests: P. Beaulé declares receiving consulting fees from MicroPort, MatOrtho, Zimmer Biomet and Corin; royalties from MicroPort, Medacta and Corin; a grant from Zimmer Biomet; and research support from DePuy and Johnson \& Johnson.

Contributors: All authors contributed substantially to the conception, writing and revision of this article and approved the final version for publication. No other competing were declared.

\section{References}

1. Rampersaud YR, Neary MA, White K. Spine adverse events severity system: content validation and interobserver reliability assessment. Spine 2010;35:790-5.

2. Millstone DB, Perruccio AV, Badley EM, et al. Factors associated with adverse events in inpatient elective spine, knee, and hip orthopaedic surgery. 7 Bone Foint Surg Am 2017;99:1365-72.
3. Chen BP, Garland K, Roffey DM, et al. Can surgeons adequately capture adverse events using the Spinal Adverse Events Severity System (SAVES) and OrthoSAVES? Clin Orthop Relat Res 2017;475:253-60.

4. Clair AJ, Inneh IA, Iorio R, et al. Can administrative data be used to analyze complications following total joint arthroplasty? $f$ Arthroplasty 2015;30:17-20.

5. Redondo-Gonzalez O, Tenias JM, Arias A, et al. Validity and reliability of administrative coded data for the identification of hospital-acquired infections: an updated systematic review with meta-analysis and meta-regression analysis. Health Serv Res 2018; 53:1919-56.

6. Lee MJ, Mohamed KMS, Kelly JC, et al. Recording adverse events following joint arthroplasty: financial implications and validation of an adverse event assessment form. F Artbroplasty 2017;32:2648-54.

7. Street JT, Thorogood NP, Cheung A, et al. Use of the Spine Adverse Events Severity System (SAVES) in patients with traumatic spinal cord injury. A comparison with institutional ICD-10 coding for the identification of acute care adverse events. Spinal Cord 2013;51:472-6.

8. Dea N, Versteeg A, Fisher C, et al. Adverse events in emergency oncological spine surgery: a prospective analysis. 7 Neurosurg Spine 2014;21:698-703.

9. Street JT, Lenehan BJ, DiPaola CP, et al. Morbidity and mortality of major adult spinal surgery. a prospective cohort analysis of 942 consecutive patients. Spine 7 2012;12:22-34.

10. Schectman JM, Plews-Ogan ML. Physician perception of hospital safety and barriers to incident reporting. 7t Comm 7 Qual Patient Saf 2006;32:337-43.

11. Rampersaud YR. CORR Insights: Can surgeons adequately capture adverse events using the Spinal Adverse Events Severity System (SAVES) and OrthoSAVES? Clin Orthop Relat Res 2017;475:261-3. 